\title{
Efficacy and safety of SOX chemotherapy with or without surgery in AFP-producing advanced gastric cancer
}

\author{
ZHU LI $^{1 *}$, XU HOU $^{1 *}$, JUAN CHEN $^{1 *}$, HUIDONG SUN $^{1}$, YUETANG MI $^{1}$, YONGLING SUI ${ }^{1}$, \\ YUHONG LI ${ }^{1}$, JIAPING XIE ${ }^{1}$, YINGLI QIAO ${ }^{1}$, XIAOFENG LEI ${ }^{1}$, XIAOSHUANG CHE ${ }^{1}$ and JUN LIU ${ }^{2}$ \\ ${ }^{1}$ Department of General Surgery, Liaocheng People's Hospital/Affiliated Liaocheng Hospital, Shandong \\ University, Liaocheng, Shandong 252000; ${ }^{2}$ Department of Liver Transplantation and Hepatobiliary Surgery, \\ Shandong Provincial Hospital Affiliated, Shandong University, Jinan, Shandong 250021, P.R. China
}

Received August 13, 2015; Accepted February 16, 2017

DOI: $10.3892 / \mathrm{ol} .2017 .6240$

\begin{abstract}
The present study investigated the clinical efficacy of S-1 plus oxaliplatin (SOX) regimen, with or without surgery in $\alpha$-fetoprotein-producing gastric cancer (APGC) with liver metastasis. A total of 24 patients with APGC treated at the Liaocheng People's Hospital between January 2011 and December 2013 were retrospectively reviewed. Clinical efficacy and patient safety were compared between the two groups. The median progression-free survival (PFS) and overall survival (OS) in the SOX group were 6.5 [95\% confidence interval (CI), 4.6-8.4] and 13.5 (95\% CI, 8.1-18.9) months, respectively. The corresponding indicators in the SOX and surgery group were 7.0 (95\% CI, 5.7-8.3) and 14 (95\% CI, 11.0-17.1) months, respectively. There was no significant difference in PFS and OS between the two groups ( $\mathrm{P}=0.703$ and 0.710 , respectively). The adverse effects of leucopenia, neutropenia, anemia and diarrhea occurred in $\sim 10 \%$ of patients in the SOX group and in $14.3 \%$ (2/14), $7.14 \%(1 / 14), 14.3 \%(2 / 14)$ and $7.14 \%(1 / 14)$, respectively, in the surgery group. No significant difference was identified between groups in terms of overall incidence of adverse effects $(\mathrm{P}=0.17)$. However, severe adverse events, including gastroplegia, pancreatic fistula, pulmonary infection and refractory ascites, occurred only in the SOX plus surgery group [incidence rate for severe adverse events, $7.14 \%$ (1/14); $\mathrm{P}<0.001$ between groups]. In conclusion, SOX chemotherapy is safe and effective in patients with APGC and liver metastasis. However, the addition of surgery to SOX
\end{abstract}

Correspondence to: Dr Zhu Li, Department of General Surgery, Liaocheng People's Hospital/Affiliated Liaocheng Hospital, Shandong University, 67 Dongchang West Road, Liaocheng, Shandong 252000, P.R. China

E-mail: 11111zhu@126.com

${ }^{*}$ Contributed equally

Key words: $\alpha$-fetoprotein-producing gastric cancer, liver metastasis, surgery, S-1 plus oxaliplatin regimen chemotherapy may not improve the disease control rate and may increase the adverse effects.

\section{Introduction}

$\alpha$-fetoprotein (AFP)-producing gastric cancer (APGC) is a rare subtype of gastric cancer that accounts for between 1.3 and $15 \%$ of all gastric cancer (GC) (1). APGC is characterized by a significant increase in serum AFP and also AFP expression in tumor cells (2). The potential underlying molecular mechanism may be the common embryonic origin of the stomach and liver from the foregut (3). The genes responsible for expression of AFP are inhibited during fetal development and may be reactivated during tumorigenesis of gastric cells $(4,5)$. APGC is associated with a poor prognosis in $<90 \%$ of patients due to its high propensity for lymphatic and venous invasion, and synchronous, as well as metachronous, liver metastases. Similar to other types of advanced GC, occurrence of complications in patients with APGC also indicates incurability. The disease is typically diagnosed at an advanced stage and is therefore associated with a high recurrence rate (6). Although surgical resection or systemic chemotherapy is used to treat liver metastases, the benefit of a palliative gastrectomy in patients with advanced GC is debatable (7). Following palliative treatment, high incidences of postoperative morbidity, prolonged hospital stay and poor quality of life have been observed frequently in patients with APGC and a poor survival rate compared with patients with non-APGC (8).

Conventional chemotherapy is predominantly ineffective in APGC. However, a previous study has suggested that a SOX (S-1 and oxaliplatin) regimen may be an effective therapy to treat advanced GC (9). S-1 is an orally active derivative of 5-fluorouracil (5-FU), which is a fourth-generation oral fluoropyrimidine (10). The study indicated that S-1-based therapy is superior compared with 5-FU in terms of overall survival (OS), progression-free survival (PFS) and objective response rate (10). For the treatment of GC, oxaliplatin is typically administered with fluorouracil and leucovorin in a combination known as FOLFOX (11). Previous studies have reported higher response rates (between 53 and 59\%) and lower toxicity with a SOX chemotherapy regimen in the treatment of advanced 
GC $(9,10,12)$. Previous phase II trials have demonstrated the superior efficacy and safety profile of the SOX regimen as a first-line treatment for advanced GC in Korean (13) and Chinese (14) patients. APGC with liver metastasis that appears to be unresectable may become resectable following treatment with neo-adjuvant chemotherapy. However, it remains controversial to perform surgery following adjuvant chemotherapy in cases of advanced gastric cancer (15). Although the use of SOX chemotherapy in gastric cancer has been established, there are limited data reported in the literature for its use, safety and efficacy to treat APGC, possibly due to its rarity. The efficacy and safety of the SOX regimen with or without resection of the primary tumor was retrospectively evaluated for the treatment of APGC.

\section{Materials and methods}

Characteristics of enrolled patients. A total of 24 patients with APGC that satisfied the inclusion and exclusion criteria were identified from Liaocheng People's Hospital (Liaocheng People's Hospital) between January 2011 and December 2013. All medical data of the patients were reviewed retrospectively and only the outcomes were updated at the time of analysis. The inclusion criteria were: i) APGC diagnosed by pathology or cytology; ii) liver metastasis diagnosed by computed tomography (CT) or magnetic resonance imaging (MRI) with >3 inoperable metastatic lesions; iii) performance status score $0-2$ according to the Eastern Cooperative Oncology Group (16); iv) no contraindication for oral medication; and v) without obvious myelosuppression including having a whole blood count of $\geq 3 \times 10^{9}$ cells $/ 1$ (normal, 4.0-10.0 cells/l), hemoglobin $\geq 8.0 \mathrm{~g} / \mathrm{dl}$ (normal 11.0-15.0 cells $/ 1$ ), platelet count of $\geq 75 \times 10^{9}$ cells $/ 1$ (normal, 100-300), adequate liver function (defined as total bilirubin $\leq 3$-fold that of the upper limit and aspartate aminotransferase/alanine aminotransferase level of $\leq 3$-fold that of the standard limit) and adequate renal function (serum creatinine $\leq 1.8 \mathrm{mg} / \mathrm{dl}$ ). The exclusion criteria included the following: i) History of severe allergy to study drugs; ii) neurological disorders or physically unable to cooperate with the present study; iii) severe dysfunction of heart, lung, liver or kidney; and iv) extensive metastasis. The present study was approved by the Ethics Committee of Liaocheng People's Hospital. Written informed consent was obtained from all study participants.

Immunohistochemistry (IHC) analysis. All of the specimens were obtained from endoscopic biopsy and surgical resection of gastric cancer tissues. The sections were treated with $3 \%$ hydrogen peroxide $\left(\mathrm{H}_{2} \mathrm{O}_{2}\right)$ and heated in microwave for antigen retrieval. Following blocking with $10 \%$ mouse serum (catalog no. M1025100, Wolcavi Biotech Co., Ltd., Beijing, China), the sections were incubated with a mouse anti-human primary antibody against AFP (1:1,000; catalog no. MAB-0013; Fuzhou Maixin Biotech Co., Ltd., Fuzhou, China) overnight at $4^{\circ} \mathrm{C}$. The sections were washed with phosphate-buffered saline (PBS, Na2HPO4 $8.1 \mathrm{mmol} / 1$, KH2PO41.5 mmol/1, NaCl 137 mmol/1, 2.7 mmol/l; PH 7.4) and then further incubated with secondary antibody (1:500, catalog no. KIT-5002; MaxVision ${ }^{\mathrm{TM}}$ HRP-Polymer anti-mouse
Table I. Characteristics of patients with APGC in the two study groups.

\begin{tabular}{|c|c|c|c|c|}
\hline Characteristic & $\begin{array}{l}\text { SOX } \\
\text { group } \\
\mathrm{n}(\%)\end{array}$ & $\begin{array}{l}\text { SOX plus } \\
\text { surgery } \\
\text { group n }(\%)\end{array}$ & $\chi^{2}$ & P-value \\
\hline Total & 10 & 14 & & \\
\hline \multicolumn{5}{|l|}{ Age, years } \\
\hline$\leq 65$ & $7(70)$ & $9(64.3)$ & 0.105 & 0.794 \\
\hline$>65$ & $3(30)$ & $5(35.7)$ & & \\
\hline \multicolumn{5}{|l|}{ Sex } \\
\hline Male & $6(60)$ & $9(64.3)$ & 2.764 & 0.486 \\
\hline Female & $4(40)$ & $5(35.7)$ & & \\
\hline \multicolumn{5}{|l|}{ PS grade } \\
\hline 0 & $2(20)$ & $2(14.3)$ & 3.079 & 0.492 \\
\hline 1 & $6(60)$ & $9(64.3)$ & & \\
\hline 2 & $2(20)$ & $3(21.4)$ & & \\
\hline \multicolumn{5}{|l|}{$\begin{array}{l}\text { Primary } \\
\text { lesion, } \mathrm{cm}\end{array}$} \\
\hline$\leq 6$ & $4(40)$ & $4(28.5)$ & 1.433 & 0.517 \\
\hline$>6$ & $6(60)$ & $10(71.5)$ & & \\
\hline \multicolumn{5}{|l|}{$\begin{array}{l}\text { Liver } \\
\text { metastasis }\end{array}$} \\
\hline$\leq 5$ & $3(30)$ & $4(28.5)$ & 2.527 & 0.479 \\
\hline$>5$ & $7(70)$ & $10(71.5)$ & & \\
\hline
\end{tabular}

APGC, $\alpha$-fetoprotein-producing gastric cancer; SOX, S-1 and oxaliplatin chemotherapy; PS, performance status.

IHC kit; Fuzhou Maixin Biotech, China). The reaction was visualized using 3,3'-diaminobenzidine (Fuzhou Maixin Biotech, China) and counterstained with Mayer's hematoxylin. A known AFP-positive slide was set as a positive control, and a slide incubated with PBS instead of primary antibody was used for a negative control. Immunostaining of AFP was mainly located in cytoplasm or membrane, presented as yellow or brown particles. Immunostaining of AFP was scored on the scale of semiquantitative assessment by combining evaluation of the intensity and percentage of positive cells. Percentage scores were assigned as follows: $0,0 \% ; 1,1-10 \% ; 2,11-50 \% ; 3,51-80 \%$; and $4,81-100 \%$. The intensity of AFP staining was scored as 0 (none), 1 (yellow), 2 (light brown) and 3 (deep brown). The scores of percentage and intensity were added to give a final score from 0 to 7 : $0-1$, negative; $2-3$, weak positive; $4-5$, positive; and above 5 , strong positive. All the slides were inspected blindly by two independent pathologists, who solved all discordant cases by discussion, or a mean value of the two scores was used.

Treatment regimen. Patients in the SOX group received only chemotherapy, whereas those in the SOX plus surgery group were treated with palliative surgical resection of the primary lesion supplemented with pre- and post-operative SOX chemotherapy. The specific regimen consisted of oxaliplatin 


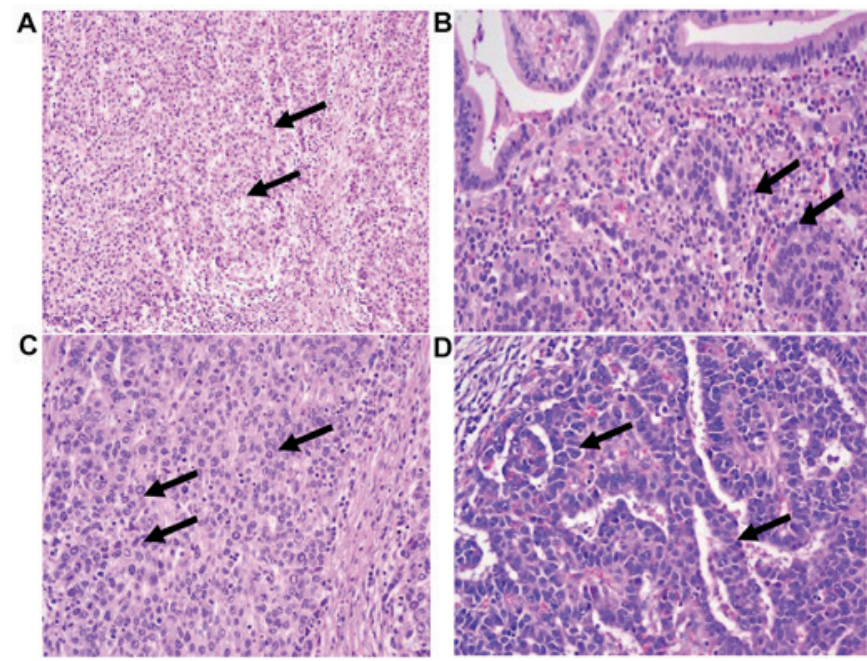

Figure 1. Histopathological examination of hematoxylin and eosin-stained $\alpha$-fetoprotein-producing gastric cancer tissues demonstrating poorly differentiated cells, diffuse growth pattern, no cell junctions, fewer glands and signet ring cells. (A) Diffusely distributed cells (magnification, x10) (B) irregular gland morphology (magnification, x100); (C) signet ring cells (magnification, x100; arrows); (D) irregular gland morphology (magnification, $\mathrm{x} 200)$.

intravenously on the first day and S-1 twice daily for 14 consecutive days. The dosage was calculated according to body surface area (BSA): BSA $<1.25 \mathrm{~m}^{2}, 80 \mathrm{mg} / \mathrm{day} ; 1.25 \mathrm{~m}^{2}$ $\leq$ BSA $<1.5 \mathrm{~m}^{2}, 100 \mathrm{mg} /$ day; BSA $>1.5 \mathrm{~m}^{2}, 120 \mathrm{mg} /$ day. This chemotherapy regime was repeated every 3 weeks with a total of $\geq 4$ cycles in the two treatment groups. Antiemetic and supportive treatments were administered according to the standard clinical practice during chemotherapy.

PFS was defined as the duration between initiation of treatment and disease progression or date of mortality or loss to follow-up. OS was defined as the duration between initiation of treatment and date of mortality or loss to follow-up. Patient status was monitored using enhanced CT, MRI, serum AFP levels and gastroscopy. The treatment efficacy following two cycles of chemotherapy was evaluated using Response Evaluation Criteria in Solid Tumors (RECIST 1.1) (17) following every two treatment cycles. Tumor lesions were measured 1 week before and 2 months after therapy. Gastroscopy was performed following chemotherapy if required. All adverse events were recorded and followed up according to the National Cancer Institute Common Terminology Criteria for Adverse Events (NCI-CTCAE4.0; version 4.03; http://evs.nci.nih. gov/ftp1/CTCAE/CTCAE_4.03_2010-06-14_QuickReference_5x7. pdf.

Statistical analysis. Statistical analyses were performed using SPSS (version 21.0; IBM SPSS, Armonk, NY, USA). The differences in clinicopathological characteristics of patients in the SOX and SOX plus surgery groups were analyzed using the $\chi^{2}$ test. PFS and OS were compared using Kaplan-Meier estimator analysis. The differences in prognoses between the two groups were analyzed using a log-rank test. Quantitative data were analyzed using Fisher's exact test. $\mathrm{P}<0.05$ was considered to indicate a statistically significant difference.
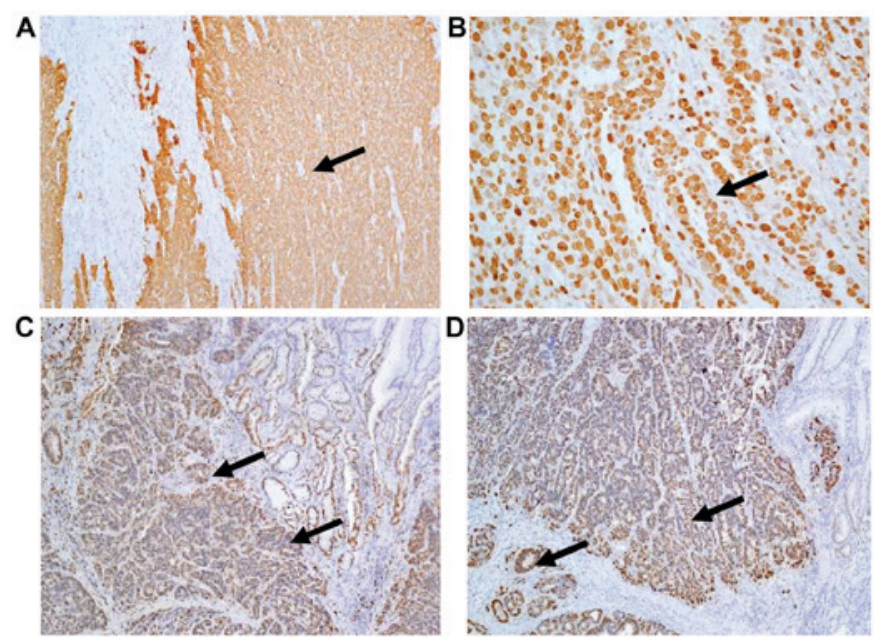

Figure 2. IHC staining of tissues of $\alpha$-fetoprotein-producing gastric cancer demonstrating positive parenchymal cancer cells (pale brown), mesenchymal (negative) cells, signet ring cells and glands (indicated by arrows). (A) Magnification, x10. (B) Magnification, x100. (C) IHC staining identifying partial cancer cell positive staining for carcinoembryonic antigen (brown). (D) IHC staining for cancer antigen 19-9 identifying cancer cells with positive staining and mesenchymal cells negative for cancer antigen 19-9 (magnification, x10). IHC, immunohistochemistry.

\section{Results}

Patient characteristics. A total of 46 patients with APGC admitted to Liaocheng People's Hospital between January 2011 and December 2013 were identified and 24 patients (15 males, 9 females; average age, 59 years, range, 32-81 years; Table I) among them met the inclusion and exclusion criteria and were enrolled in the present retrospective study. A total of 10 patients were assigned to the SOX group as they were resistant to docetaxel, cisplatin and/or 5-FU. In total, 14 patients were assigned to the SOX plus surgery group and underwent SOX chemotherapy prior to and following palliative resection of the primary lesion. All patients exhibited typical histopathological characteristics, including poorly differentiated adenocarcinoma and signet ring cells (Fig. 1). AFP was detected using immunohistochemical staining (stained in brown; Fig. 2A and $\mathrm{B}$ ); carcinoembryonic antigen and cancer antigen 125 were also positive (Fig. 2C and D). Typical characteristics of GC were observed upon gastroscopy. However, the lesions of certain patients were comparatively small and were frequently less ulcerated (Fig. 3). All patients exhibited significantly elevated AFP levels. Patients with metastatic lesions of $>5 \mathrm{~cm}$ in diameter exhibited AFP levels $>3,000 \mu \mathrm{g} / 1$.

Efficacy evaluation. All patients were monitored using CT during and following treatment, including alterations in the largest horizontal diameter of the tumor multiplied by the largest vertical diameter, the number of tumors and any newly diagnosed tumors (Figs. 4-6). In the SOX group, $4(40 \%)$ cases exhibited a partial response; in $3(30 \%)$ cases, the patient's condition was evaluated as stable and $3(30 \%)$ cases demonstrated evidence of disease progression. In the SOX plus surgery group, $6(42.8 \%)$ cases exhibited a partial response, 4 (28.6\%) cases remained stable and 4 (28.6\%) cases demonstrated evidence of disease progression. The disease 

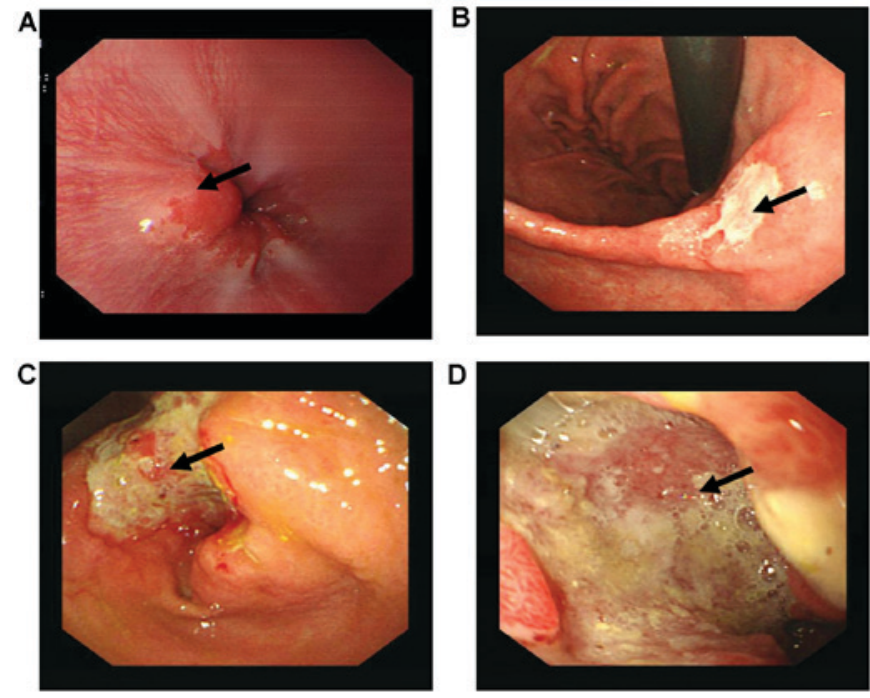

Figure 3. Images of APGC lesions captured from gastroscopy. (A) Patient with erosive gastritis. (B) APGC with a small focus (lesion diameter, $1 \mathrm{~cm}$ ). (C) APGC with medium focus (diameter, $<6 \mathrm{~cm}$ ). (D) APGC with a large focus (diameter, $>6 \mathrm{~cm}$ ). Arrows indicate (A) gastritis and (B-D) APGC lesions. APGC, $\alpha$-fetoprotein-producing gastric cancer.

Table II. Comparison of treatment efficacy in the two study groups.

\begin{tabular}{lcc}
\hline Outcome & $\begin{array}{c}\text { SOX group } \\
\mathrm{n}(\%)\end{array}$ & $\begin{array}{c}\text { SOX plus } \\
\text { surgery group } \\
\mathrm{n}(\%)\end{array}$ \\
\hline $\mathrm{CR}$ & 0 & 0 \\
$\mathrm{PR}$ & $4(40)$ & $6(42.8)$ \\
$\mathrm{SD}$ & $3(30)$ & $4(28.6)$ \\
$\mathrm{PD}$ & $3(30)$ & $4(28.6)$ \\
DCR & $7(70)$ & $4(71.4)$ \\
PFS, months & $6.5(4.6-8.4)$ & $7.0(5.7-8.3)$ \\
OS, months & $13.5(8.1-18.9)$ & $14(11.0-17.1)$ \\
\hline
\end{tabular}

SOX, S-1 and oxaliplatin chemotherapy; CR, cure rate; PR, partial response; $\mathrm{SD}$, stable disease; $\mathrm{PD}$, progressed disease; $\mathrm{DCR}$, disease control rate; PFS, progression-free survival; OS, overall survival.

control rate was 70.0 and $71.4 \%$ in the SOX and SOX plus surgery group, respectively. However, no statistically significant difference was identified ( $\mathrm{P}=0.208$; Table II).

The dynamics of the plasma AFP levels were monitored in patients during and following treatment. AFP detection in plasma was identified as a sensitive indicator of APCG treatment efficacy (Fig. 7).

The PFS in the two groups was 6.5 [95\% confidence interval (CI), 4.6-8.4] and 7 (95\% CI, 5.7-8.3) months, respectively. The observed difference between groups was not significant ( $\mathrm{P}=0.703$; Table II; Fig. 8A). The OS of the two groups was 13.5 (95\% CI, 8.1-18.9) and 14 (95\% CI, 11.0-17.1) months, respectively. No statistically significant difference between groups was identified ( $\mathrm{P}=0.710$; Table II; Fig. 8B).
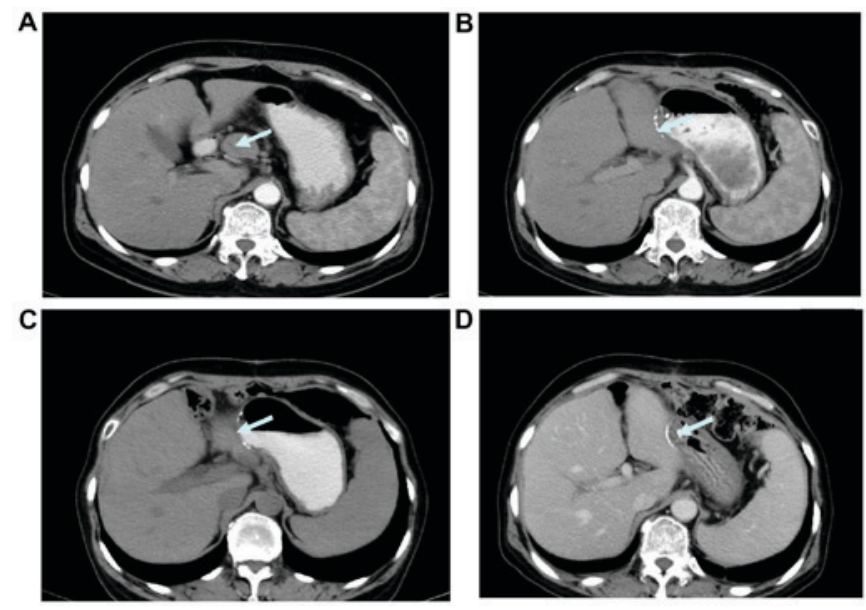

Figure 4. CT radiographs of $\alpha$-fetoprotein-producing gastric cancer patient (SOX plus surgery group) with extragastric lymphatic metastasis treated with radical gastrectomy (distal subtotal gastrectomy and D2 lymph node dissection) and post-operative SOX regimen for 6 cycles. (A) Extragastric metastatic lymph nodes. (B) CT scan 2 months after surgery. (C) CT scan a year following surgery. (D) CT scan 2 years after surgery demonstrating no signs of recurrence. (A) Arrows indicate extragastric metastatic lymph nodes. (B-D) Titanium nail sutures in the stump of stomach are indicated by arrows. SOX, S-1 and oxaliplatin chemotherapy regime; CT, computed tomography.
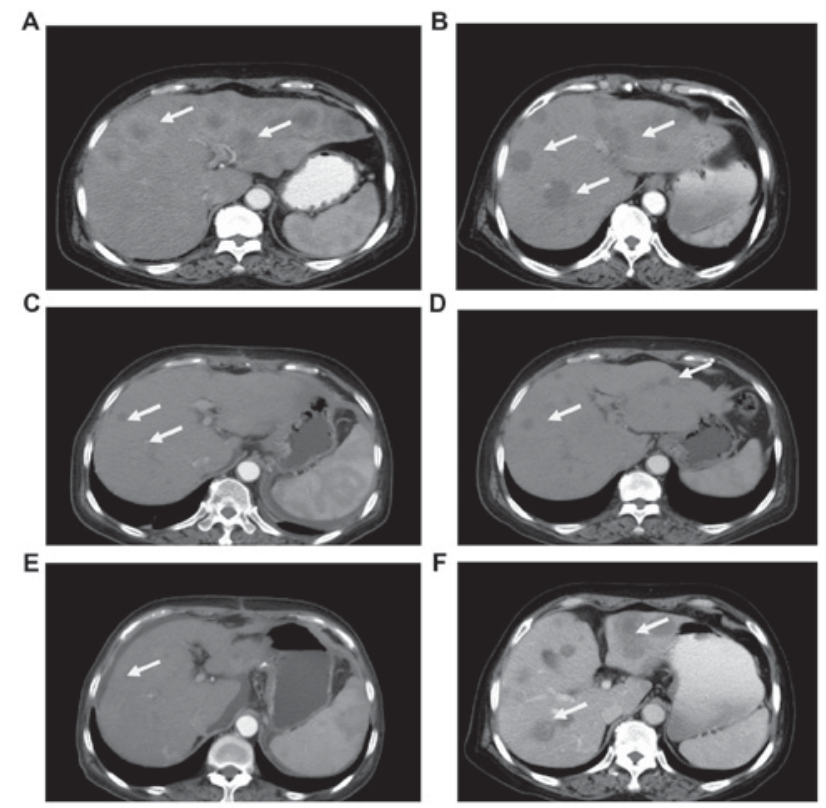

Figure 5. Computed tomography radiograph of a patient with $\alpha$-fetoprotein-producing gastric cancer treated with SOX plus subtotal gastrectomy. (A) Multiple hepatic metastatic lesions at enrollment. (B) Attenuated metastatic lesions with smaller margin of tumor invasion following a month of SOX. (C) Reduced metastases following 3 months of chemotherapy. (D) Flared-up metastatic lesions caused by interruption in chemotherapy due to recurrent fever in the 2 months following surgery. (E) Attenuated metastatic lesions following SOX chemotherapy started 4 months after surgery. (F) Recurrence of metastatic lesions 1 year after surgery due to interruption in chemotherapy necessitated by the poor general condition of the patient. The patient developed multiple metastases 1 year after surgery and succumbed to multiple organ failure 6 months later. SOX, S-1 and oxaliplatin chemotherapy. Arrows indicate extragastric metastatic lymph nodes.

Safety profile. Adverse events in the study population are summarized in Table III. Level 3 to 4 adverse effects included leucopenia, granulocytopenia, anemia and diarrhea with 
Table III. Comparison of adverse effects between SOX and SOX plus surgery groups.

\begin{tabular}{|c|c|c|c|c|}
\hline \multirow[b]{2}{*}{ Adverse effect } & \multicolumn{2}{|c|}{ SOX group n $(\%)$} & \multicolumn{2}{|c|}{ SOX plus surgery group n (\%) } \\
\hline & Grade 1-2 & Grade 3-4 & Grade 1-2 & Grade 3-4 \\
\hline \multicolumn{5}{|l|}{ Hematology } \\
\hline Leucopenia & $4(40.0)$ & $1(10.0)$ & $6(42.9)$ & $2(14.3)$ \\
\hline Granulocytopenia & $5(50.0)$ & $1(10.0)$ & $6(42.9)$ & $1(7.1)$ \\
\hline Thrombocytopenia & $4(40.0)$ & 0 & $5(35.7)$ & 0 \\
\hline Anemia & $5(50.0)$ & $1(10.0)$ & $8(57.1)$ & $2(14.3)$ \\
\hline \multicolumn{5}{|l|}{ Non-hematological } \\
\hline Nausea & $2(20.0)$ & 0 & $3(21.4)$ & 0 \\
\hline Vomiting & $4(40.0)$ & 0 & $6(42.9)$ & 0 \\
\hline Diarrhea & $4(40.0)$ & $1(10.0)$ & $5(35.7)$ & $1(7.1)$ \\
\hline Constipation & $1(10.0)$ & 0 & $1(7.1)$ & 0 \\
\hline Anorexia & $6(60.0)$ & 0 & $9(64.3)$ & 0 \\
\hline Hypodynamia & $6(60.0)$ & 0 & $8(57.1)$ & 0 \\
\hline Peripheral neuropathy & $3(30.0)$ & 0 & $4(28.6)$ & 0 \\
\hline
\end{tabular}

SOX, S-1 and oxaliplatin chemotherapy.
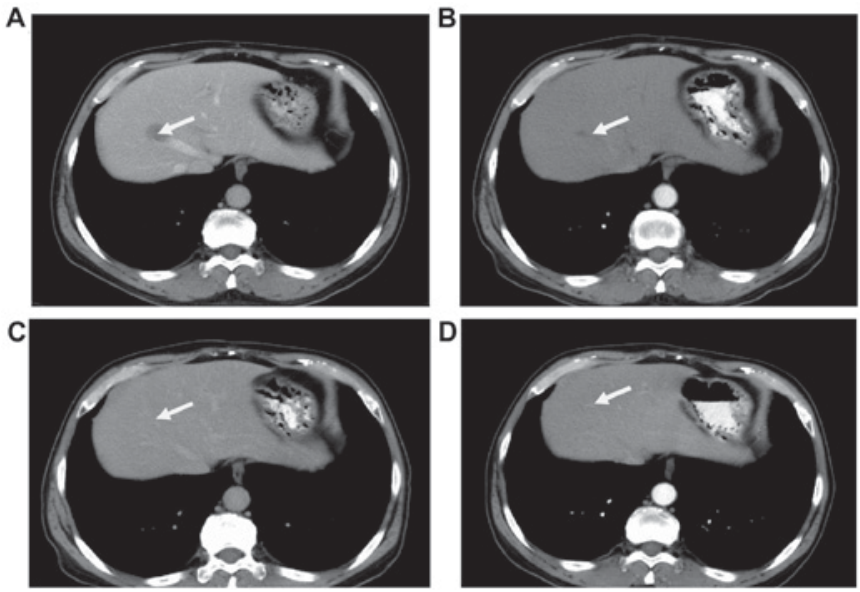

Figure 6. Computed tomography radiographs of a patient with $\alpha$-fetoprotein-producing gastric cancer treated with SOX. (A) A metastatic lesion in liver at enrollment. (B) Significantly attenuated metastatic lesion in the liver following 1 month of SOX treatment. (C) No sign of metastasis following two months of SOX treatment. (D) No recurrence of metastasis following 2 years of regular SOX regimen and without surgical resection of primary lesion. SOX, S-1 and oxaliplatin chemotherapy. Arrows indicate intrahepatic metastatic lesions.

no significant difference identified between the two groups $(\mathrm{P}>0.05)$. In the SOX plus surgery group, 1 case of each surgery-associated event (gastroplegia, pancreatic fistula, pulmonary infection and refractory ascites) was reported. However, all patients improved with expectant treatment with no treatment-associated mortality.

\section{Discussion}

GC is the fourth most frequent cancer globally (18). In total, $84 \%$ of patients with GC develop advanced disease, with a median survival without chemotherapy of between 3 and 4 months. For advanced-stage GC, chemotherapy is the preferred treatment option (19). Samaratunga et al have suggested that neoadjuvant chemotherapy with at least two cycles of the SOX regimen may induce a relatively high number of pathologically complete responses without increasing morbidity and mortality (20). Consequently, the SOX regimen is considered to be one of the most effective chemotherapy treatments for GC, particularly in China and Japan (it is not available in the majority of other countries) (9-12). The SOX regimen is now considered as a chemotherapeutic option for the treatment of GC. However, its efficacy in patients with APGC has not been conclusively demonstrated.

Elevated serum AFP levels are frequently observed in patients with hepatocellular carcinoma and yolk sac tumors (21). According to certain studies, AFP may be produced by gastrointestinal tract organs, rectal carcinoma, gallbladder carcinoma and lung carcinoma (22). Bourreille et al (21) reported the first case of APGC with liver metastasis in 1970 . Liu et al $(1,23)$ reported $>100$ cases of patients with APGC since 1970. APGC is established to be an aggressive tumor with a higher propensity for liver metastasis. APGC is associated with immunosuppression due to increased levels of AFP expression and a poorer prognosis as compared with other subtypes of GC (24). Key factors affecting the prognosis of APGC include primary lesion progression, histopathological characteristics of cancer cells and immune status of the patient (21).

As the precise underlying mechanism of APGC remains to be elucidated, the optimal treatment approach requires further consideration. Patients with APGC and liver metastasis may be eligible for resection of the primary if neo-adjuvant chemotherapy is administered. Studies have suggested that obstruction, perforation or bleeding may be 


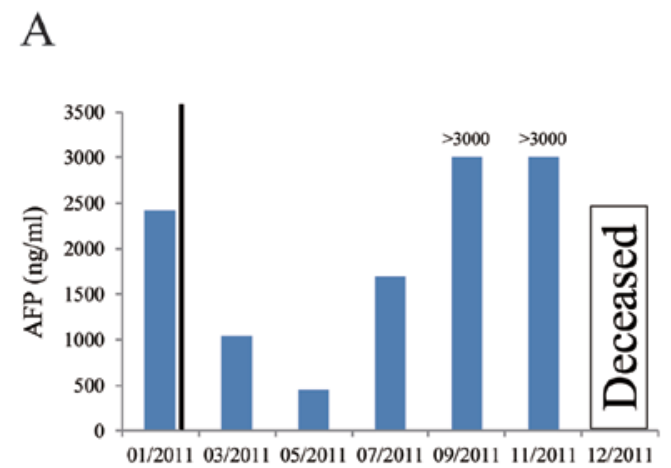

Month, year

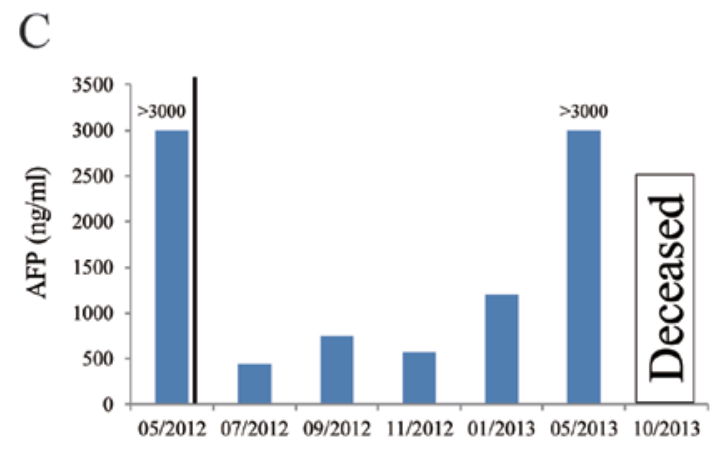

Month, year

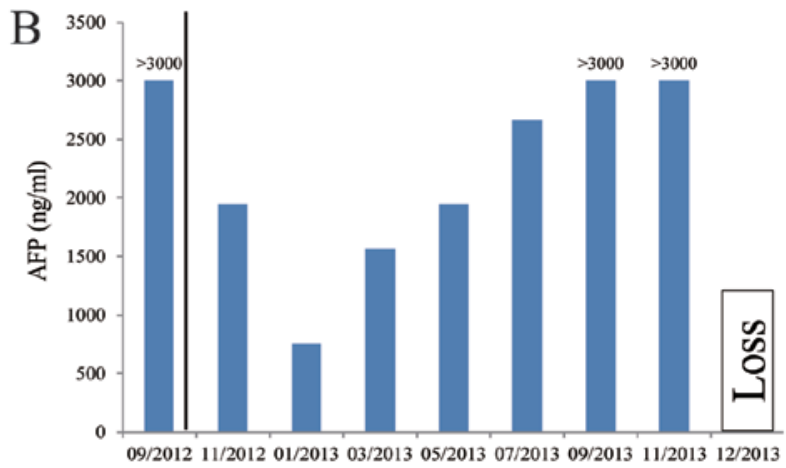

Month, year

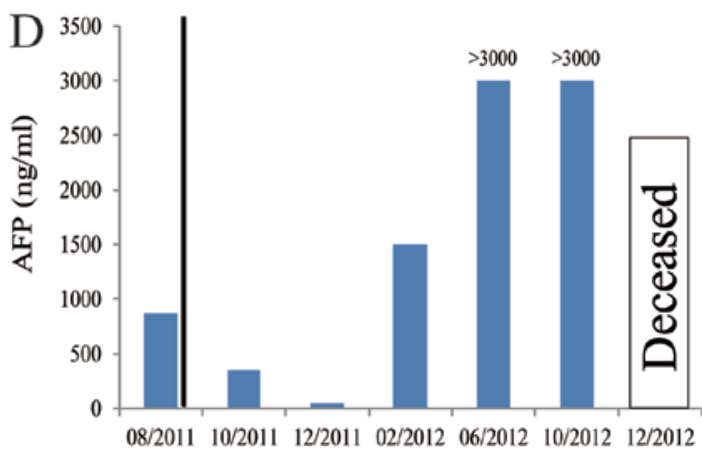

Month, year

Figure 7. Temporal representation of AFP levels in AFP-producing gastric cancer. (A) Typical trend of AFP levels of a patient in the SOX group. (B) Typical trend of AFP levels of a patient in the SOX group. (C) Typical trend of AFP levels of a patient in the SOX plus surgery group. (D) Typical trend of AFP levels of a patient in the SOX plus surgery group. Black lines indicate starting points for treatment. AFP, $\alpha$-fetoprotein; SOX, combination chemotherapy with S-1 and oxaliplatin; deceased, succumbed to disease; loss, loss to follow-up.
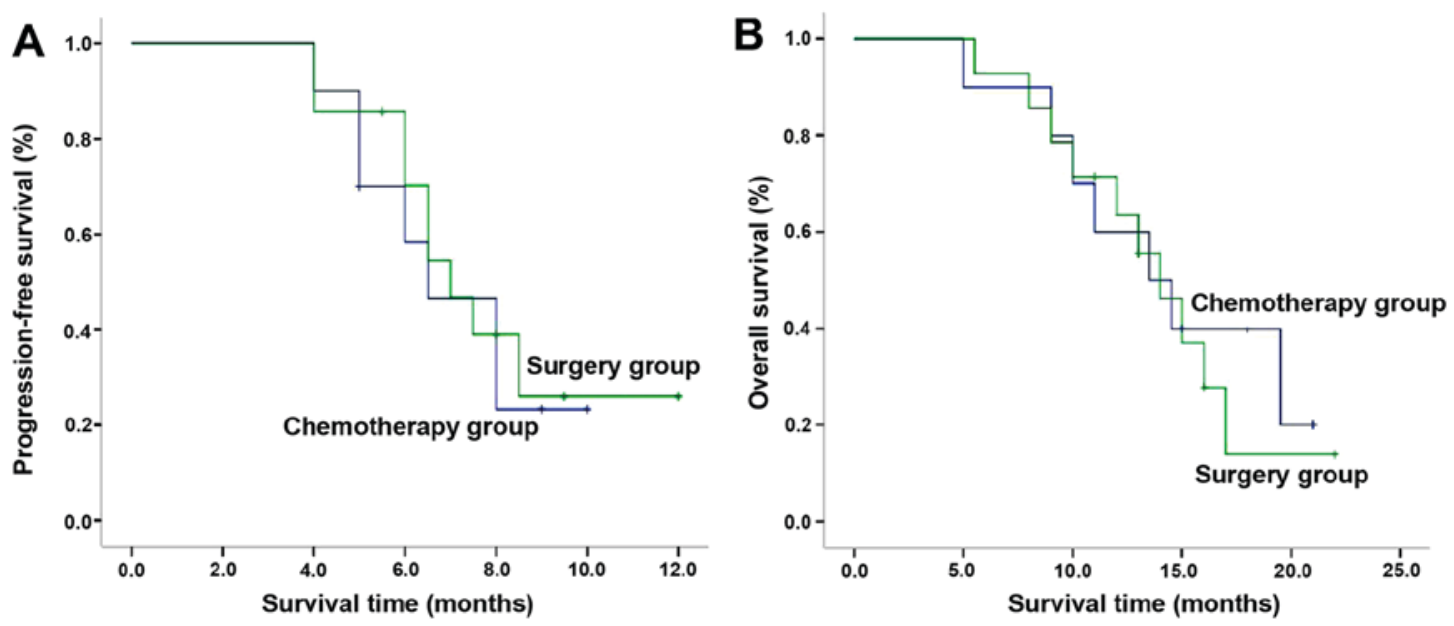

Figure 8. Kaplan-Meier estimator survival curves. (A) Progression-free survival rate in the two study groups. (B) Overall survival rate in the two groups. Chemotherapy group, SOX treatment alone; Surgery group, SOX treatment plus surgery (resection of primary lesion). SOX, S-1 and oxaliplatin chemotherapy.

eliminated when the primary tumor is resected in patients with advanced APGC and multiple organ metastases (25-27). It may also decrease tumor burden and the energy demand of patients. Studies have also demonstrated a decrease in cytokine levels and significant immunosuppression in these patients (24). Patients with APGC and liver metastasis exhibit an average survival time of $\leq 14$ months despite treatment with adjuvant therapy (14). It has been suggested that percutaneous ethanol injection may be an effective treatment for liver metastasis and is associated with an average survival time of 18 months, which is longer than that associated with other treatment modalities, including partial hepatic resection and systemic chemotherapy (the latter having a survival time of $\leq 7$ months) (8). Another previous study initially proposed that 
patients with a solitary liver metastatic lesion should be treated with surgical resection, whereas patients with multiple liver metastases should undergo a partial hepatectomy followed by transarterial continuous-infusion chemotherapy as the preferred therapeutic approach (28). Notably, all patients in this study did not survive for $>8$ months, demonstrating that hepatic resection should be considered carefully (24). In the present study, the efficacy and safety profile of SOX chemotherapy alone was retrospectively compared with the combined approach of SOX plus surgical resection in patients with APGC.

The efficacy of the SOX regimen to treat APGC was positive in the present study compared with previous studies involving patients with other types of GC, and those treatments had a significantly lower efficacy (9-11). The majority of metastatic lesions were decreased to half of their original size following one cycle of chemotherapy (Figs. 4-6). The plasma AFP level also decreased, but the same response was not observed in the primary tumor in the stomach using gastroscopy. The present study investigated whether unresectable primary tumors may become resectable through the administration of neo-adjuvant chemotherapy. The surgery was performed following between 2 and 3 cycles of chemotherapy, or following significant reduction in metastatic lesions and decreased AFP levels. Serious complications were more frequently observed in the SOX plus surgery group, including fever of unknown origin, pulmonary infection, pancreatic fistula and refractory ascites. The complications were associated with decreased tolerability of further chemotherapy, which in the majority of cases was delayed by $>3$ months after surgery. This delay resulted in disease progression in certain cases and was possibly responsible for increased drug resistance in these patients. The patients who exhibited resistance to drugs typically did not survive due to complications within 1 year of surgery. The results of the present study suggest that the advantage that may be gained by palliative resection of the primary tumor may be offset by the disadvantage from chemotherapy interruption. The palliative surgery may be performed following the administration of planned chemotherapy cycles or following the attainment of plateau response to chemotherapy.

In the SOX group, 4 patients demonstrated a partial response to treatment, 3 cases remained in a stable condition and 3 cases exhibited evidence of disease progression. In the SOX plus surgery group, 6 cases demonstrated a partial response, 4 cases remained stable and in 4 cases there was evidence of disease progression. The disease control rate was 70.0 and $71.4 \%$ in SOX and SOX plus surgery group, respectively, with no significant difference identified between the two groups $(\mathrm{P}=0.208)$, which suggested that resection of the primary lesion may not lower the tumor burden or affect metastatic lesions. The PFS in the SOX and SOX plus surgery groups was 6.5 (95\% CI, 4.6-8.4) and 7 (95\% CI, 5.7-8.3) months, respectively. The corresponding OS was 13.5 (95\% CI, 8.1-18.9) and 14 (95\% CI, 11.0-17.1) months, respectively. No significant difference in OS was identified between the two groups $(\mathrm{P}=0.710)$. These results suggested that there is no additional benefit from surgery to the PFS or OS rate. Additionally, primary lesion surgery did not appear to confer any prognostic advantage on patients with APGC and liver metastasis. The results of the present study differ from those of certain earlier studies $(29,30)$, possibly as all subjects in the present study were patients with APGC.

The adverse events rates in the two groups were comparable. However, only patients in the SOX plus surgery group experienced severe adverse events, including gastroplegia, pancreatic fistula, pulmonary infection and refractory ascites. Although adjuvant chemotherapy prior to surgery aided in the surgical resection of the primary lesion, the post-surgical complications affected the continuity of chemotherapy leading to tumor resistance to chemotherapy. This phenomenon may contribute to the decreased efficacy of surgery observed in the present study. A robust evaluation of the SOX plus surgery regimen in patients with APGC is required.

In conclusion, the results of the present single-center retrospective study demonstrates the specific efficacy of SOX regimen in patients with APGC and provide evidence that irregular chemotherapy with SOX may lead to the development of tumor resistance. Future randomized, double-blind, large-scale clinical trials are required to conclusively establish the role of the SOX regimen in patients with APGC.

\section{References}

1. Liu X, Cheng Y, Sheng W, Lu H, Xu Y, Long Z, Zhu H and Wang Y: Clinicopathologic features and prognostic factors in alpha-fetoprotein-producing gastric cancers: Analysis of 104 cases. J Surg Oncol 102: 249-255, 2010.

2. Chun $\mathrm{H}$ and Kwon SJ: Clinicopathological characteristics of alpha-fetoprotein-producing gastric cancer. J Gastric Cancer 11: 23-30, 2011

3. Ooi A, Nakanishi I, Sakamoto N, Tsukada Y, Takahashi Y, Minamoto T and Mai M: Alpha-fetoprotein (AFP)-producing gastric carcinoma. Is it hepatoid differentiation? Cancer 65: 1741-1747, 1990.

4. Jia Y, Liu D, Xiao D, Ma X, Han S, Zheng Y, Sun S, Zhang M, Gao H, Cui X and Wang Y: Expression of AFP and STAT3 is involved in arsenic trioxide-induced apoptosis and inhibition of proliferation in AFP-producing gastric cancer cells. PLoS One 8: e 54774, 2013.

5. Liu DP, He QJ and Liu CG: Correlations among Helicobacter pylori infection and the expression of cyclooxygenase- 2 and vascular endothelial growth factor in gastric mucosa with intestinal metaplasia or dysplasia. J Gastroenterol Hepatol 25: 795-799, 2010

6. Kim T and Yu W: Prognostic value of preoperative serum alphafetoprotein level in resectable gastric cancer. J Korean Gastric Cancer Assoc 3: 33-37, 2003 (In Korean).

7. Lim JH, Lee DH, Shin CM, Kim N, Park YS, Jung HC and Song IS: Clinicopathological features and surgical safety of gastric cancer in elderly patients. J Korean Med Sci 29: 1639-1645, 2014.

8. Sato Y, Nishimaki T, Date K, Shirai Y, Kurosaki I, Saito Y, Watanabe T and Hatakeyama K: Successful resection of metachronous liver metastasis from alpha-fetoprotein-producing gastric cancer: Report of a case. Surgery Today 29: 1075-1088, 1999.

9. Zhong DT, Wu RP, Wang XL, Huang XB, Lin MX, Lan YQ and Chen Q: Combination chemotherapy with S-1 and oxaliplatin (SOX) as first-line treatment in elderly patients with advanced gastric cancer. Pathol Oncol Res 21: 867-873, 2015.

10. Liu GF, Tang D, Li P, Wang S, Xu YX, Long AH, Zhou NL, Zhang LL, Chen J and Xiang XX: S-1-based combination therapy vs S-1 monotherapy in advanced gastric cancer: A meta-analysis. World J Gastroenterol 20: 310-318, 2014.

11. Shi C, Chen Q, Shen S, Wu R, Yang B, Liu Q and Xu Q: Paclitaxel combined with oxaliplatin as first-line chemotherapy for locally advanced or metastatic gastric cancer. Expert Rev Anticancer Ther 15: 595-601, 2015.

12. Yang L, Yang YI, Qin Q, Zhou A, Zhao J, Wang J, Shu C, Yuan X and Hu S: Evaluation of the optimal dosage of S-1 in adjuvant SOX chemotherapy for gastric cancer. Oncol Lett 9: 1451-1457, 2015. 
13. Oh SY, Kwon HC, Jeong SH, Joo YT, Lee YJ, Cho Sh, Kang MH, Go SI, Lee GW, Kim Hg and Kang JH: A phase II study of S-1 and oxaliplatin (SOx) combination chemotherapy as a first-line therapy for patients with advanced gastric cancer. Invest New Drugs 30: 350-356, 2012.

14. Liu B, Ying J, Luo C, Xu Q, Zhu L and Zhong H: S-1 combined with oxaliplatin as first line chemotherapy for Chinese advanced gastric cancer patients. Hepatogastroenterology 59: 649, 2012.

15. Nishikawa K, Fujitani K, Inagaki H, Akamaru Y, Tokunaga $S$, Takagi M, Tamura S, Sugimoto N, Shigematsu T, Yoshikawa T, et al: Randomised phase III trial of second-line irinotecan plus cisplatin versus irinotecan alone in patients with advanced gastric cancer refractory to S-1 monotherapy: TRICS trial. Eur J Cancer 51: 808-816, 2015.

16. Oken MM, Creech RH, Tormey DC, Horton J, Davis TE, McFadden ET and Carbone PP: Toxicity and response criteria of the Eastern Cooperative Oncology Group. Am J Clin Oncol 5: 649-655, 1982

17. Eisenhauer EA, Therasse P, Bogaerts J, Schwartz LH, Sargent D, Ford R, Dancey J, Arbuck S, Gwyther S, Mooney M, et al: New response evaluation criteria in solid tumours: Revised RECIST guideline (version 1.1). Eur J Cancer 45: 228-247, 2009.

18. Ferlay J, Soerjomataram I, Dikshit R, Eser S, Mathers C, Rebelo M, Parkin DM, Forman D and Bray F: Cancer incidence and mortality worldwide: Sources, methods and major patterns in GLOBOCAN 2012. Int J Cancer 136: E359-E386, 2015.

19. Feng D, Leong M, Li T, Chen L and Li T: Surgical outcomes in patients with locally advanced gastric cancer treated with S-1 and oxaliplatin as neoadjuvant chemotherapy. World J Surg Oncol 13: 11, 2015.

20. Samaratunga H, Samaratunga D, Dunglison N, Perry-Keene J, Nicklin J and Delahunt B: Alpha-fetoprotein-producing carcinoma of the renal pelvis exhibiting hepatoid and urothelial differentiation. Anticancer Res 32: 4987-4991, 2012.

21. Bourreille J, Metayer P, Sauger F, Matray F and Fondimare A: Existence of alpha feto protein during gastric-origin secondary cancer of the liver. Presse Med 78: 1277-1278, 1970 (In French).

22. Lunghi A, Petreni P, Romanelli RG, Vizzutti F, Marra F, Tarquini R and Laffi G: Aggressive gastric carcinoma producing alpha-fetoprotein: A case report and review of the literature. Case Rep Oncol 7: 92-96, 2014.
23. Liu X, Yang M, Gao J, Zhang S and Xi Y: Clinicopathologic features and prognosis of 51 patients with $\alpha$-fetoprotein-producing gastric cancer. Zhonghua Zhong Liu Za Zhi 37: 231-234, 2015 (In Chinese)

24. Yamashita T, Nakane A, Watanabe T, Miyoshi I and Kasai N: Evidence that alpha-fetoprotein suppresses the immunological function in transgenic mice. Biochem Biophys Res Commun 201: $1154-1159,1994$

25. Iida M, Imura J, Furuichi T, Sawada T, Nagawa H and Fujimori T: Alteration of the AT motif binding factor- 1 expression in alpha-fetoprotein producing gastric cancer: Is it an event for differentiation and proliferation of the tumors? Oncol Rep 11: 3-7, 2004.

26. Tiberio GA, Roviello F, Donini A and de Manzoni G; Italian Research Group for Gastric Cancer: Hepatic metastases from gastric cancer: A surgical perspective. World J Gastroenterol 21: 11489-11492, 2015

27. Kerkar SP, Kemp CD and Avital I: Liver resections in metastatic gastric cancer. HPB (Oxford) 12: 589-596, 2010.

28. Liu SF, Lu CR, Cheng HD, Xi HQ, Cui JX, Li JY, Shen WS and Chen L: Comparison of therapeutic efficacy between gastrectomy with transarterial chemoembolization plus systemic chemotherapy and systemic chemotherapy alone in gastric cancer with synchronous liver metastasis. Chin Med J (Engl) 128: 2194-2201, 2015.

29. Li Z, Fan B, Shan F, Tang L, Bu Z, Wu A, Zhang L, Wu X, Zong X, Li S, et al: Gastrectomy in comprehensive treatment of advanced gastric cancer with synchronous liver metastasis: A prospectively comparative study. World J Surg Oncol 13: 212, 2015.

30. Kinoshita T, Kinoshita T, Saiura A, Esaki M, Sakamoto H and Yamanaka T: Multicentre analysis of long-term outcome after surgical resection for gastric cancer liver metastases. Br J Surg 102: 102-107, 2015. 